\title{
Ionization Chamber of New Conception for Enviromental Gas Radon Measurements
}

\author{
V. Mossa ${ }^{1,3, *}$, G. Roselli ${ }^{2}$, C. Pastore ${ }^{1}$, V. Paticchio ${ }^{1}$, L. Vitucci ${ }^{2}$ \\ ${ }^{1}$ INFN, sez. Bari, Via Orabona 4, 70125 Bari, Italy. \\ ${ }^{2}$ ARPA Puglia DAP Bari, U.O.S. Polo di Specializzazione Radiazioni Ionizzanti, Via Oberban 18/E, 70126 Bari, Italy. \\ ${ }^{3}$ Università degli Studi di Foggia, Via Napoli 25, 71122 Foggia, Italy.
}

How to cite this paper: V. Mossa, G. Roselli, C. Pastore, V. Paticchio, L. Vitucci. (2021) Ionization Chamber of New Conception for Enviromental Gas Radon Measurements. International Journal of Clinical and Experimental Medicine Research, 5(2), 185-191. DOI: 10.26855/ijcemr.2021.04.012

Received: February 28, 2021

Accepted: March 26, 2021

Published: April 15, 2021

*Corresponding author: V. Mossa, NFN, sez. Bari, Via Orabona 4, 70125 Bari, Italy; Università degli Studi di Foggia, Via Napoli 25, 71122 Foggia, Italy.

Email: viviana.mossa@ba.infn.it

\begin{abstract}
Long exposure to high radon levels leads to an increase of developing lung cancer risk, due to irradiation of lung tissue by the $\alpha$ particles emitted by radon and its decay products. Currently radon is considered to be the second cause of lung cancer, after only cigarettes smoke. Another very interesting aspect related to the radon concentration is its potential use as a seismic events precursor, according to some geophysical models that suggest the release of radon from the underground as a result of tectonic deformation responsible for the earthquake. The detector evaluated in this study is an ionizing chamber, operating in free air at atmospheric pressure. It consists of a metal cylinder used as a cathode and a wire anode placed along the axis. It provides radon concentration measurements with a fast time response, useful for sudden changes in radon gas emission. The prototype tested and discussed in this paper is extremely compact, economical, with a long autonomy of operation, easy to install and use. A new version of the detector has been developed and characterized, with a cylindrical steel cathode, having a mesh structure. The detection performances are similar to the previous prototype with direct operation into the atmosphere and reduced weight, size and energy consumption. The detector presented could be used in a widespread network on the territory, in order to evaluate the radon gas concentration in living environments and/or to detect radon emission before a seismic event.
\end{abstract}

\section{Keywords}

Ionizing Chambers, Radon Gas Detector, Health Safety, Lung Cancer, Hearth Quake Precursor

\section{Introduction}

Radon is a radioactive gas naturally found in three isotopes: ${ }^{222} \mathrm{Rn},{ }^{219} \mathrm{Rn},{ }^{220} \mathrm{Rn}$ and produced respectively by radioactive series of ${ }^{238} \mathrm{U},{ }^{235} \mathrm{U}$ and ${ }^{232} \mathrm{Th}$. Of the three, only the ${ }^{222} \mathrm{Rn}$ isotope has a half-life long enough $\left[\mathrm{T}_{1 / 2}\left({ }^{222} \mathrm{Rn}\right)\right.$ $=3.82$ days, $\left.\mathrm{T}_{1 / 2}\left({ }^{220} \mathrm{Rn}\right)=55.6 \mathrm{~s}, \mathrm{~T}_{1 / 2}\left({ }^{219} \mathrm{Rn}\right)=3.96 \mathrm{~s}\right]$ to allow its release from the soil and the rocks where it is generated.

Outdoors it disperses quickly, but indoors (homes, schools, workplaces, etc.) it can accumulate, reaching in some cases high concentrations. The consequence of prolonged exposure to high levels of radon is an increased risk of developing lung cancer, due to irradiation of lung tissue by the $\alpha$ particles emitted by radon and its decay products (Figure 1) [1].

Another very interesting aspect related to anomalous radon concentration is its potential use as a seismic events 
precursor, according to some geophysical models that suggest that the release of radon from the underground is a result of tectonic deformation that causes the earthquake [2] [3]. This study requires the use of a huge number of fast detectors operating online. Several commercial detectors can meet this demand, but they are usually too expensive to be employed in a massive application because of their excellent performances. For this purpose, however, it is sufficient to record simultaneously signals, thus providing a reliable information about Rn accumulation.

In this paper, two different gas detectors have been tested for radon concentration measurements: both are cylindrical ionization chambers, but, the former presents a standard configuration, the second, instead, is characterized by a mesh cathode.

\section{Materials and methods}

The experimental set up is shown in Figure 2.

The standard ionization chamber prototype is an air-filled detector operating at atmospheric pressure, with two electrodes (anode and cathode). The electrodes are in the form of a cylinder arrangement with a coaxially located internal anode wire (Figure 3). All tests were performed under stable environmental conditions of humidity ( 40\%) and temperature $\left(\sim 22^{\circ} \mathrm{C}\right)$.

In a cylindrical counter with an inner and an outer radius a and $\mathrm{b}(\mathrm{a}<<\mathrm{b})$ respectively, the potential difference is given by:

where:

$$
V=-\frac{Q}{l C_{l}}\left[\frac{m^{2} \mathrm{Kg}}{C s^{2}}\right]
$$

- The capacitance per unit length $C_{l}$ is defined as $\frac{2 \pi \varepsilon}{\ln \left(\frac{b}{a}\right)}\left[\frac{F}{m}\right]$,

- The total charge $Q$ created within the detector is equal to $\frac{E e}{W}[C]$, with $\mathrm{E}=5 \mathrm{MeV}$ the incident radiation energy, $\mathrm{W}=33.9 \mathrm{eV}$ the average energy loss per ion pair produced in the gas and $\mathrm{l}[\mathrm{m}]$ the chamber length.

From equation (1), the total potential difference depends only on the produced charge, once the ionization chamber dimensions have been set. The technical characteristics of the present detector are summarized in Table 1.

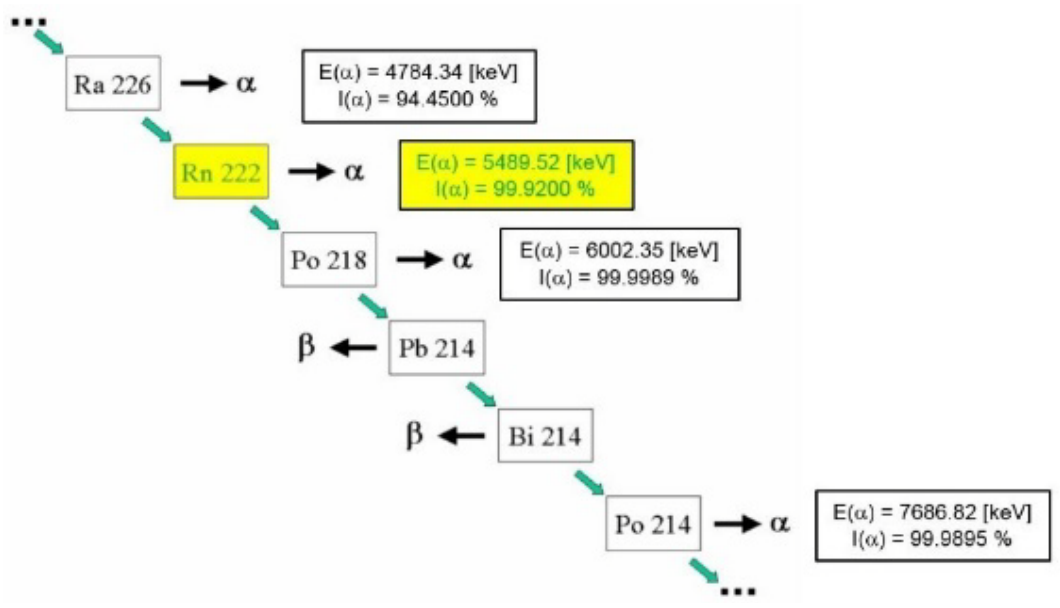

Figure 1. Part of the decay chain of ${ }^{238} \mathrm{U}$ from where ${ }^{222} \mathrm{Rn}$ originates.

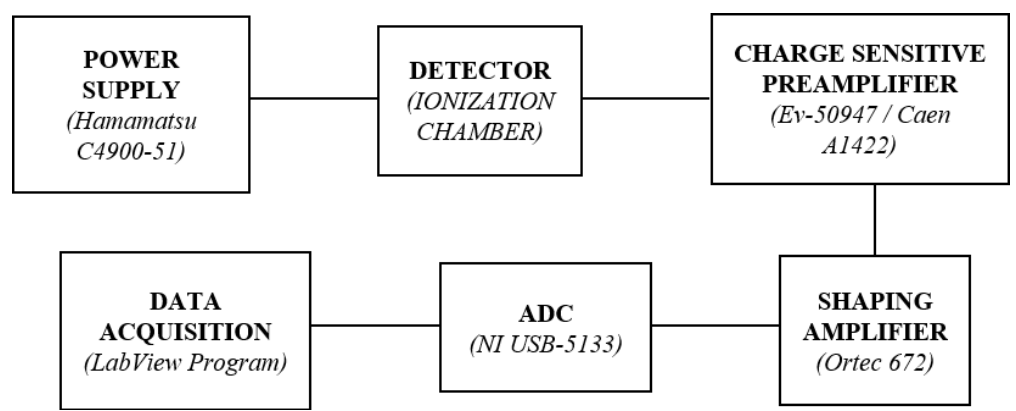

Figure 2. Experimental set up block diagram. 


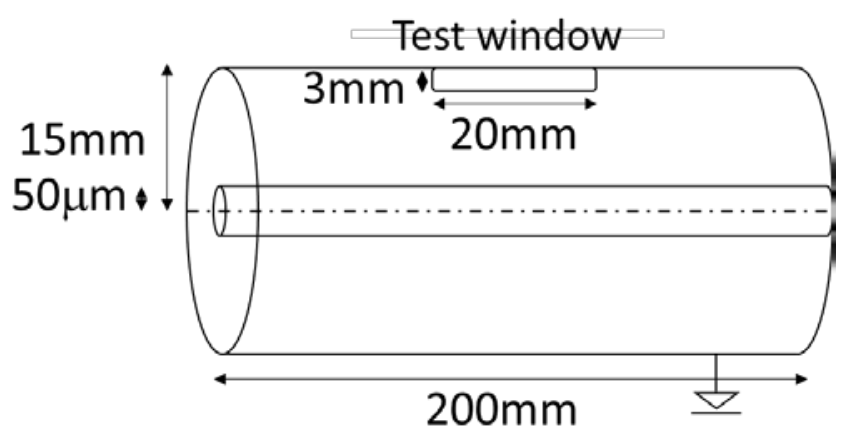

Figure 3. A schematic drawing of the standard ionization chamber.

Table 1. Technical detector characteristics

\begin{tabular}{ccc}
\hline $\mathbf{C}_{\mathbf{l}}$ & $9.75 \mathrm{pF} / \mathrm{m}$ \\
\hline $\mathbf{Q}$ & $2.36 \cdot 10^{14} \mathrm{C}$ \\
$\mathbf{V}$ & $12 \mathrm{mV}$ \\
\hline
\end{tabular}

\section{Preliminary study and results}

Similary to Charpak et al. method, developed in [4] [5] [6], a preliminary test with $\alpha$ particles at $5.5 \mathrm{MeV}$ of energy was performed, using a ${ }^{241}$ Amsource of known activityin order to evaluate the detector voltage characteristic. The amplitude of the observed pulse from the detector plotted versus the voltage applied to the electrodes is shown in Figure 4. The flat region in the trend identifies the voltage values of the ionization chamber operating mode.

The final evaluation of the detector was performed directly with ${ }^{222} \mathrm{Rn}$ gaseous source of known activity (Pylon Model RNC: $0.083 \mathrm{kBq} \pm 4 \%$ ). The radon was generated via ${ }^{226} \mathrm{Ra}$ decay in a metallic box containing small amount of dry powder. Figure 5 shows a schematic drawing of the experimental apparatus, where the pump is used to extract the radon gas from the source and to inject it into the circuit.

First, a background measurement was performed in order to evaluate the presence of environmental radon and electronic noise, finding a mean value $\mathrm{N}_{\mathrm{B}}=0.001 \mathrm{cps}$, thus the number of counts/sec produced by the alpha source should be calculated taking into account this background counting rate.

Then, the measurements were performed with gaseous source turning on the pump system and regulating the flow to 1 litre/minute, at a tension value of $800 \mathrm{~V}$.

The absolute efficiency of the ionization chamber to alpha particles can be defined as the ratio of the number of pulses recorded $\mathrm{N}$ and the number of the radiation quanta emitted by the source $\mathrm{A}_{\text {det }}$. The former can be calculated by subtracting the background contribution to the number of counts per second detected with the radioactive source $N_{s}$, while the latteris defined as $\mathrm{A}_{\text {det }}=\mathrm{A} \cdot \frac{v_{\text {det }}}{v_{\text {tot }}}$, where $\mathrm{A}$ is the nominal source activity and $v_{\text {det }}$ and $v_{\text {tot }} \cdot{ }^{1}$ are respectively the detector and the total system volumes:

$$
\varepsilon=\frac{N}{A_{\text {det }}}=\frac{N_{s}-N_{B}}{A_{\text {det }}}=0.0101 \pm 0.0014
$$

Once evaluated the detection efficiency, it is possible to calculate the chamber limits of detectability (Table 2):

- the Minimum Detectable Activity (MDA), according to the Currie equation [8]

$$
M D A=\frac{4.65 \cdot \sqrt{N_{B}}}{t \cdot \varepsilon}
$$

with $\mathrm{t}=$ measurements time;

- the Minimum Detectable Concentration (MDC) defined as

$$
M D C=\frac{M D A}{v_{\text {det }}}
$$

\footnotetext{
1 The total system volume is calculated as $v_{\text {tot }}=v_{\text {detector }}+v_{\text {source }}+v_{\text {tubes }}$.
} 


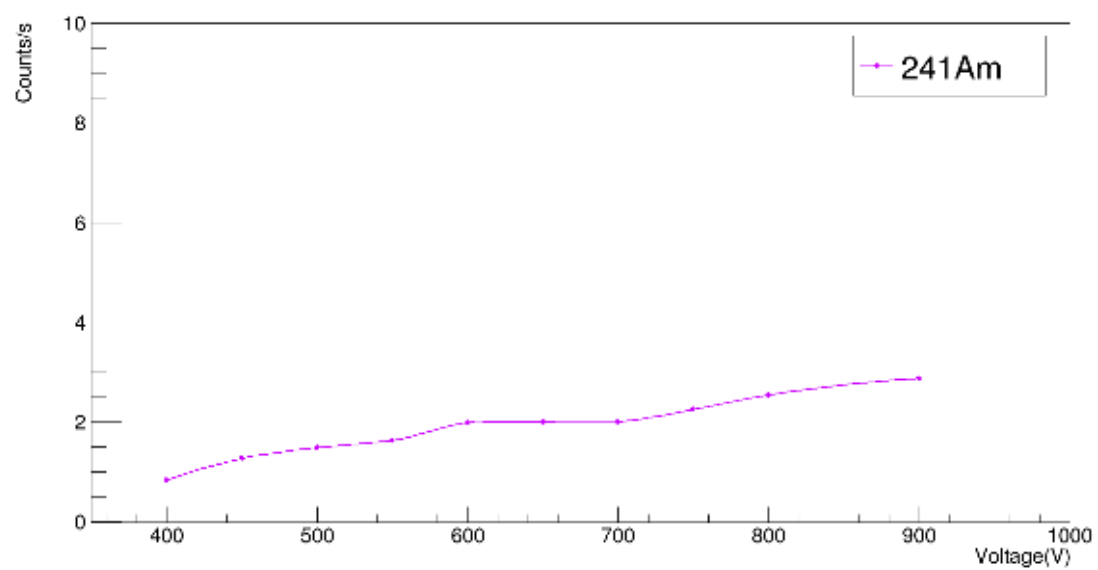

Figure 4. Detector voltage characteristic [7].

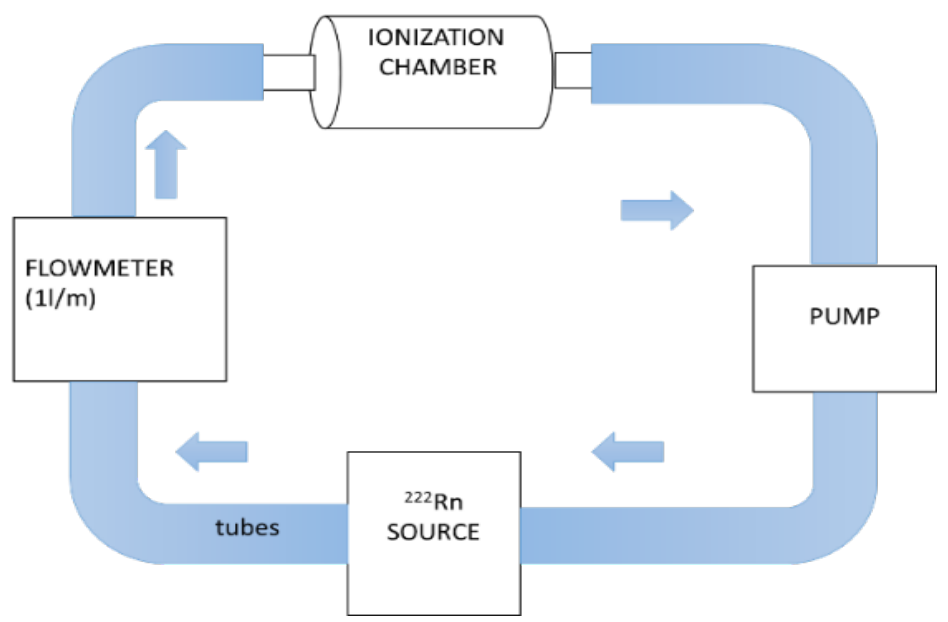

Figure 5. Schematic drawing of the experimental setup based on the standard ionization chamber.

Table 2. Chamber limits of detectability

\begin{tabular}{cccc}
\hline$\varepsilon(\%)$ & MDA (Bq) & MDC $\left(\mathrm{Bq} / \mathrm{m}^{3}\right)$ \\
\hline $1 \pm 0.1$ & 0.04 & 6,642 \\
\hline
\end{tabular}

Equation (4) allows the time need for detecting known radon concentrations to be evaluated. In fact,

$$
M D C=\frac{4.65 \sqrt{\frac{N_{B}}{T_{B}} \cdot T_{M}}}{\varepsilon \cdot T_{M} \cdot v_{\text {det }}} \rightarrow T_{M}=\left(\frac{4.65 \sqrt{\frac{N_{B}}{T_{B}}}}{\varepsilon \cdot M D C \cdot v_{\text {det }}}\right)^{2}
$$

where $T_{M}$ and $T_{B}$ indicate respectively the measurement time and the background acquisition time. With respect to radon concentration limits for indoor places provided by the regulations in force [9], the detector will spend about 8 days to reveal $300 \mathrm{~Bq} / \mathrm{m}^{3}$ concentration and about 2 months and a half for $100 \mathrm{~Bq} / \mathrm{m}^{3}$ concentration (Figure 6).

Based on the obtained results, a new prototype of ionization chamber has been built and characterized (Figure 7). It has a cylindrical mesh-structured cathode. The detection performances (Table 3) are similar to the previous prototype (the electric field acting on the alpha particle is almost the same), with direct operation into the atmosphere (without air injection) and reduced weight, size and energy consumption.

Table 3. Technical detector characteristics

\begin{tabular}{cc}
\hline $\mathbf{C}_{\mathbf{l}}$ & $9.75 \mathrm{pF} / \mathrm{m}$ \\
\hline $\mathbf{Q}$ & $2.36 \cdot 10^{14} \mathrm{C}$ \\
$\mathbf{V}$ & $48 \mathrm{mV}$ \\
\hline
\end{tabular}


The experimental procedure adopted for studying and characterizing the detector is similar to the previous one:

1. Preliminary study with ${ }^{241} \mathrm{Am}$ source for the detector voltage characterization (Figure 8);

2. Detector characterization with a calibrated gas ${ }^{222} \mathrm{Rn}$ source. For this purpose, the sensor has been immersed in $\mathrm{a}{ }^{222} \mathrm{Rn}$ atmosphere, by flowing the gas in a chamber having a total volume of about 91 , as shown in Figure 9 .

The analysis results, expressed in terms of detection efficiency and limits of detectability are summarized in Table 4:

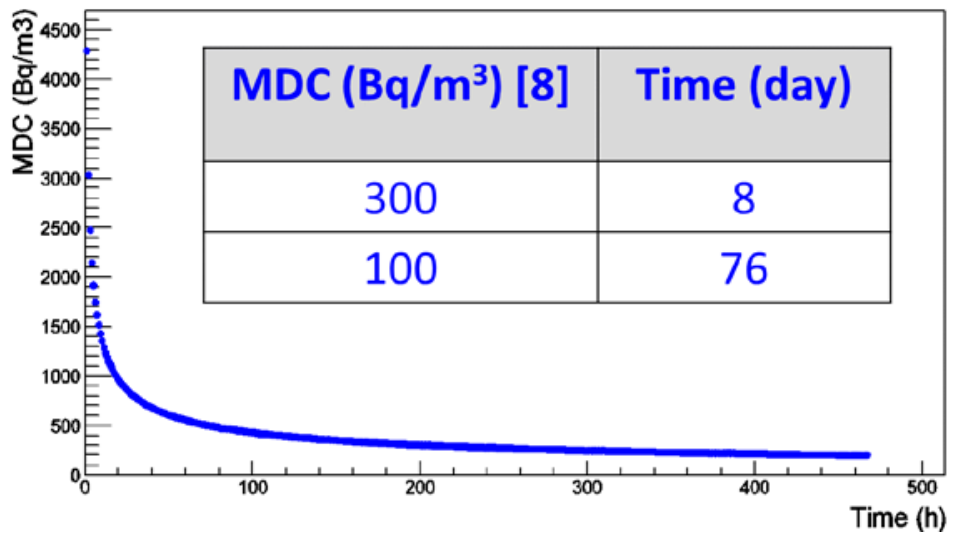

Figure 6. Ionization chamber Minimum Detectable Concentration vs acquisition time [7].

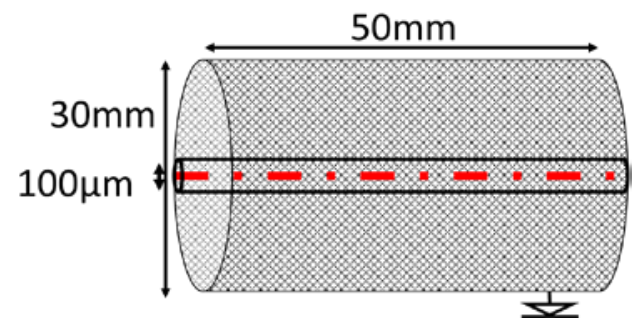

Figure 7. New prototype schematic drawing.

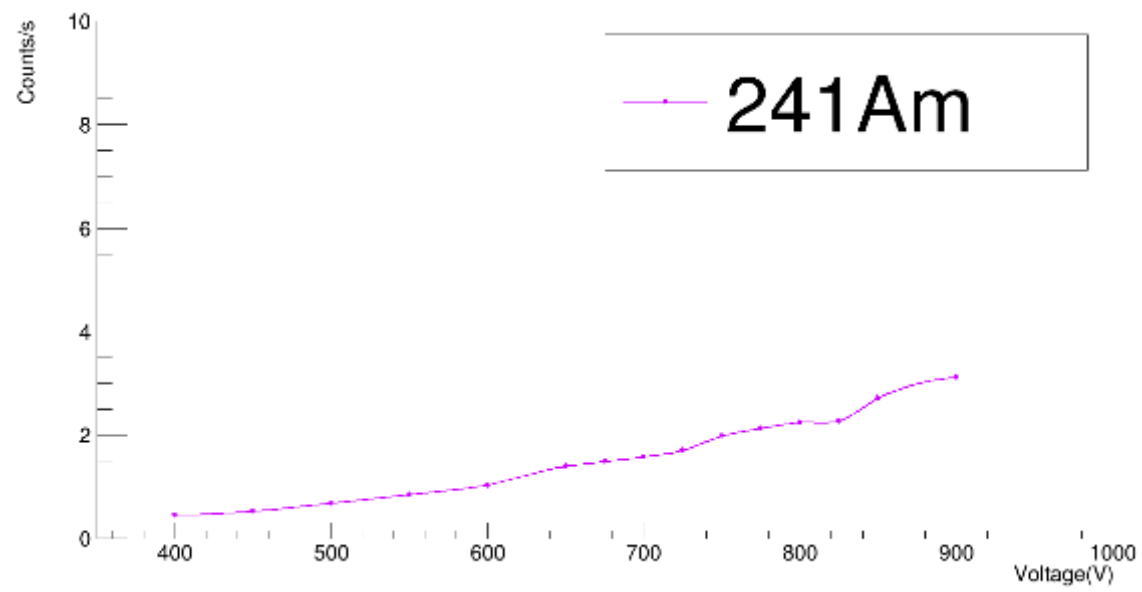

Figure 8. Voltage characteristic of the mesh-structurated ionization chamber.

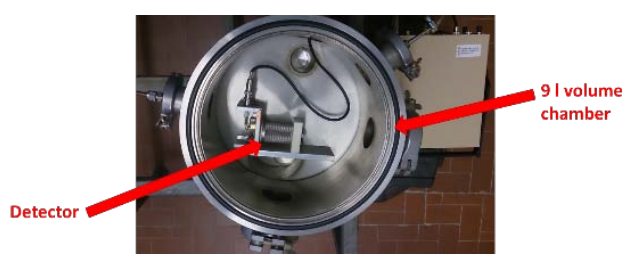

Figure 9. New prototype experimental setup. 
Table 4. Chamber limits of detectability

\begin{tabular}{ccc}
\hline$\varepsilon(\%)$ & MAR $(\mathrm{Bq})$ & $\mathrm{MDC}\left(\mathrm{Bq} / \mathrm{m}^{3}\right)$ \\
\hline $6.73 \pm 0.5$ & 0.04 & $1,204.41$ \\
\hline
\end{tabular}

Finally, the study of the minimum detectable concentration as a function of time has produced the results shown in Figure 10: the detector will spend about 3 days for measuring a $300 \mathrm{~Bq} / \mathrm{m}^{3}$ radon concentration and about one month for $100 \mathrm{~Bq} / \mathrm{m}^{3}$ concentration.

Similary to the tests performed by Charpak et al. in [5], a temporal response of the detection system has been studied. The test is implemented as follows:

- Gas flow in the active volume of the detector for a well-defined time interval;

- Chamber purge by flushing clean air (pumping speed is unchanged).

The Figure 11 and Figure 12 show the trend of the counting rate versus the exposure time with and without source for the first and the second chamber prototype respectively.

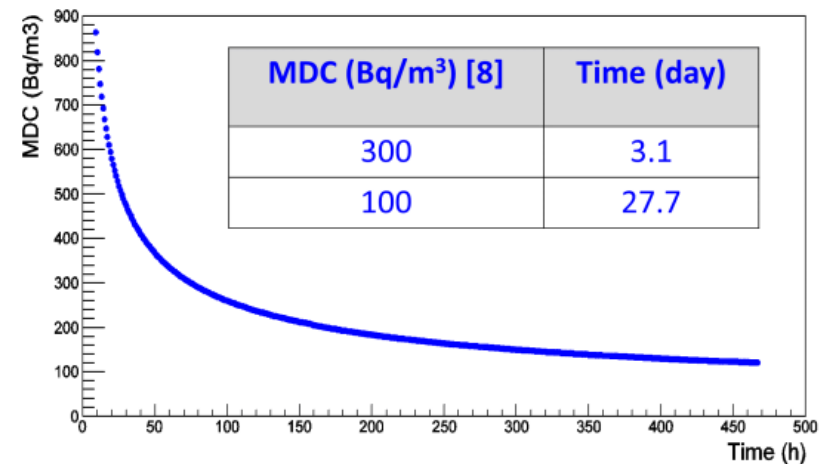

Figure 10. New prototype Minimum Detectable Concentration vs acquisition time.

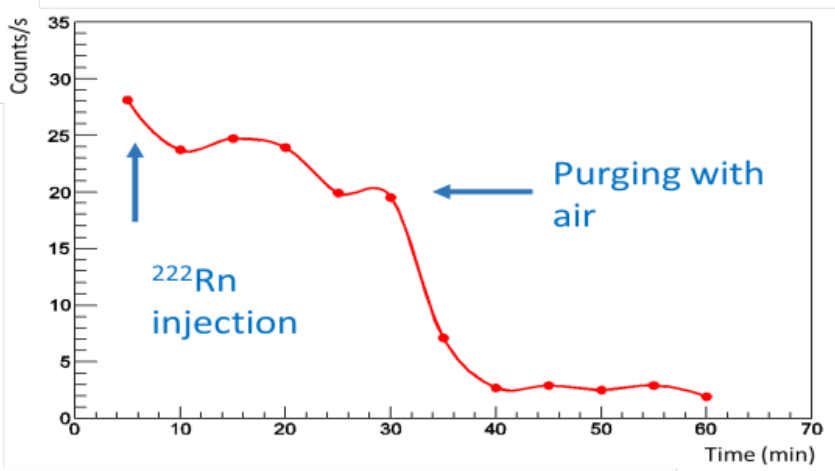

Figure 11. Counting rate versus time (logarithmic scale), for the standard ionization chamber [7].

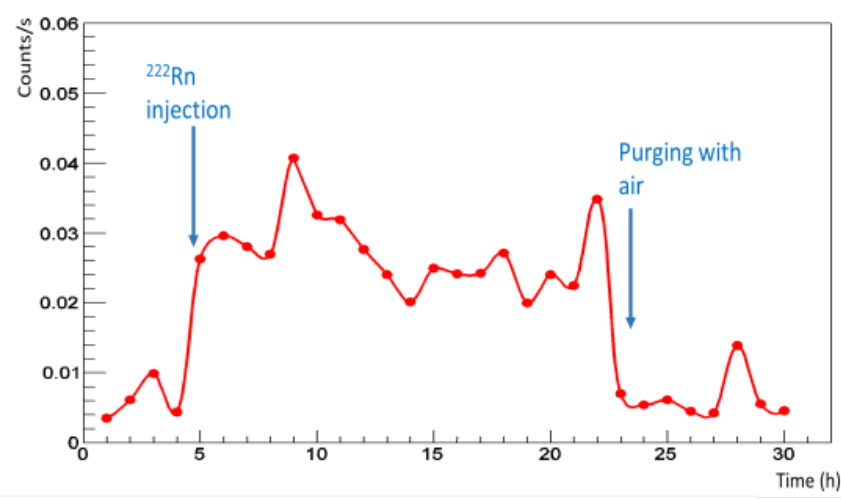

Figure 12. Counting rate versus time (linear scale), for the mesh-structurated ionization chamber. 


\section{Conclusions}

The obtained results allow the system performances to be quantified and the possible practical applications for the radon measurement to be identified.

The ionization chamber can provide a quick response in function of time, useful for monitoring sudden changes in radon concentration. Furthermore, the detector is extremely compact, economical, with a long enough autonomy of operation, easy to install and use. Hence, this detector could be potentially used in a widespread network on the territory for the radon gas concentration measurement in living environments and/or aimed at testing theoretical models which establish a correlation between radon emission and seismic event.

\section{References}

[1] Gaskin, J., et al. (2018). Global Estimate of Lung Cancer Mortality Attributable to Residential Radon. Environmental Health Prespectives, 126(5): 057009.

[2] Tareen, A. D. K., et al. (2019). Automated anomalous behaviour detection in soil radon gas prior to earthquakes using computational intelligence techniques. Journal of Environmental Radioactivity, 203: 48-54.

[3] Tareen, A. D. K., et al. (2019). Descriptive analysis and earthquake prediction using boxplot interpretation of soil radon time series data. Applied Radiation and Isotopes, 154: 108861.

[4] Charpack, G., et al. (2008). Detector of alpha particles and x-rays operating in ambient air in pulse counting mode or/and with gas amplification, JINST 3 P02006.

[5] Charpak, G., et al. (2010). Performance of wire-type Rn detectors operated with gas gain in ambient air in view of its possible application to early earthquake predictions. arXiv:1002.4732.

[6] Charpak, G., et al. (2011). Results from prototypes of environmental and health alarm devices based on gaseous detectors operating in air in counting mode. Nucl. Instrum. and MethodsPhys. Res., A 628.

[7] Mossa, V. (2013). Caratterizzazione di una camera a ionizzazione per misure di radon ambientale e confront con tecniche convenzionali, Master Thesis in Physics, Università degli Studi di Bari “Aldo Moro” (unpublished thesis).

[8] Currie, L. (1968). Limits for Qualitative Detection and Quantitative Determination. Anal.Chem., 40: 3.

[9] Council Directive 2013/59/EURATOM, December 5, 2013, Official Journal of the European Union L13/1 (art. 54-74). 